\title{
Transgender youth: current concepts
}

\section{Stephen M. Rosenthal, MD}

Division of Pediatric Endocrinology, Child and Adolescent Gender Center, Benioff Children's Hospital, University of California San Francisco, San Francisco, CA, USA
Received: 7 November, 2016

Accepted: 9 December, 2016

Address for correspondence: Stephen M. Rosenthal, MD

Division of Pediatric Endocrinology, Child and Adolescent Gender Center, Benioff Children's Hospital, University of California San Francisco, Mission Hall, 550 -16th Street, 4th Floor, San Francisco, CA, USA

Tel: +1-415-476-2266

Fax: +1-415-476-5356

E-mail: Stephen.Rosenthal@ucsf.edu
In many countries throughout the world, increasing numbers of gender nonconforming/transgender youth are seeking medical services to enable the development of physical characteristics consistent with their experienced gender. Such medical services include use of agents to block endogenous puberty at Tanner stage II with subsequent use of cross-sex hormones, and are based on longitudinal studies demonstrating that those individuals who were first identified as gender dysphoric in early or middle childhood and continue to meet the mental health criteria for being transgender at early puberty are likely to be transgender as adults. This review addresses terms and definitions applicable to gender nonconforming youth, studies that shed light on the biologic determinants of gender identity, current clinical practice guidelines for transgender youth, challenges to optimal care, and priorities for research.

Keywords: Transgender, Gender nonconforming, Gender dysphoria, Gonadotropin releasing hormone agonist, Cross-sex hormones

\section{Definitions, prevalence, and mental health concerns}

While a person's "sex" refers to the physical attributes that characterize biologic maleness or femaleness (e.g., the genitalia), "gender identity" refers to a person's inner sense of self as male or female and is not always binary. At birth, most children are assigned a "sex of rearing" based on genital anatomy. With that assignment comes societal expectations of gender roles, behaviors, and expressions, which are sometimes referred to as a child's "assigned (or natal) gender $^{\text {"1) }}$. Despite the practice of "assigning" a gender in the newborn period, it is important to note that gender identity can only be assumed, and not, in fact, known until an individual achieves a particular level of psychological development and self-awareness. An individual for whom gender identity and physical sex characteristics are in alignment is sometimes referred to as "cisgender."

"Gender nonconforming" is a term sometimes used when gender identity is not consistent with the gender assumed based on birth sex assignment ${ }^{1)}$. The term "Transgender" has been used to describe a person who transiently or persistently identifies with a gender different from their "natal gender" ${ }^{\prime 2}$. The World Professional Association for Transgender Health (WPATH) Standards of Care (SOC) describes "transgender" (adjective) as "a diverse group of individuals who cross or transcend culturally defined categories of gender" and transsexual" (adjective) as "individuals who seek to change or have changed their primary and/or secondary sex characteristics through feminizing or masculinizing medical interventions (hormones and/ or surgery), typically accompanied by a permanent change in gender role."3). "Transsexual" has also been used to describe a person who identifies as a member of the gender opposite to that assigned at birth ${ }^{4,5}$, but who has not necessarily sought medical and/or surgical interventions.

"Transgender," defined more narrowly, refers to an individual whose gender identity is the "opposite" of their "assigned" or "natal gender." A person with a female gender identity and male assigned sex would be referred to as a "transgender girl/woman," "transfemale," or MTF (male to female); a person with a male gender identity and female assigned sex would be 
referred to as a "transgender boy/man," "transmale," or FTM").

"Gender behavior" is not equivalent to gender identity, and the majority of youth with gender non-conforming behavior will not turn out to have a transgender identity ${ }^{4,5,7)}$. In the Diagnostic and Statistical Manual of Mental Disorders (DSM) 5, "Gender dysphoria" refers to clinically significant distress (of at least 6 month's duration) related to the incongruence between one's "affirmed" or "experienced gender" and one's "assigned (or natal) gender." This term replaces "Gender Identity Disorder" (GID) found in the earlier DSM IV. Replacing "disorder" with "dysphoria" underscores the concept that a transgender identity is no longer considered pathological, and instead, focuses on dysphoria as the clinical concern ${ }^{2}$.

"Sexual identity" (or "sexual orientation") is often confused with "gender identity." While the former refers to whom one is sexually attracted, the latter refers to whom one "is" as male, female, or somewhere on the gender continuum. Any individual, whether transgender or cisgender, may have any sexual orientation.

The prevalence of transgender youth is not known. However, multidisciplinary clinics for transgender youth and adolescents in Europe and North America and some parts of Asia (personal communication) have seen a steadily increasing demand for services in recent years ${ }^{9-12}$. Studies in adults suggest that the prevalence of being transgender may be as high as 1 in 200 individuals ${ }^{13)}$.

Regarding mental health considerations, transgender youth/ young adults had a 2- to 3-fold increased risk of anxiety, depression, suicidal ideation, suicide attempts, and self-harm without lethal intent in comparison to controls ${ }^{14)}$. The impact of the degree of parental support on mental health outcomes was reported in transgender youth and young adults age 16 to 24 years $(n=84)$ from Ontario, Canada: Satisfaction with life and self-esteem were significantly greater in transgender youth whose parents were "very supportive" vs. those whose parents were "somewhat to not at all supportive" ${ }^{15)}$. In addition, depression and suicide attempts were significantly decreased in transgender youth whose parents were supportive in comparison to those whose parents were not supportive ${ }^{15}$.

\section{Biological determinants of gender identity}

Numerous studies from a variety of biomedical disciplinesendocrine, genetic, and neuroanatomical-have begun to shed light on the biological determinants of gender identity ${ }^{16)}$. Results of these studies support the concept that gender identity is not simply a psychosocial construct, but likely reflects a complex interplay of biologic, environmental, and cultural factors.

While the majority of transgender patients do not have a disorder/difference of sex development (DSD), studies of gender identity outcome within the endocrine discipline have been principally carried out in patients with a variety of DSDs, primarily exploring the role of prenatal and postnatal androgens in gender identity development. For example, in studies of
46, XX patients with "classical" congenital adrenal hyperplasia (CAH) caused by mutations in the CYP21A2 gene resulting in 21-hydroxylase deficiency and varying degrees of genital masculinization, there was a greater than expected number of patients with gender dysphoria, "atypical gender identity", or who were transgender ${ }^{17-19)}$. In a meta-analysis of 250 patients with 46, XX 21-hydroxylase deficiency leading to virilizing $\mathrm{CAH}$ and raised female, nearly $95 \%$ of patients reported a female gender identity without gender dysphoria, while 5.2\% reported either a male gender identity or gender dysphoria ${ }^{18}$. Of note, there was no apparent correlation with the degree of genital masculinization and gender identity outcome ${ }^{18}$. This and other studies demonstrate that the majority of 46 , XX patients with virilizing $\mathrm{CAH}$ from 21-hydroylase deficiency appear to have a female gender identity. However, the finding that $5.2 \%-11.6 \%$ of such patients have gender dysphoria, an "atypical gender identity" or are transgender would appear to be much more common than expected based on the reported prevalence of FTM transsexualism, implying that there is some role for prenatal/ postnatal androgens in gender identity outcome ${ }^{17-19)}$. It is noteworthy that in $46, \mathrm{XX}$ individuals with virilizing $\mathrm{CAH}$ from 21-hydroylase deficiency, prenatal androgens are more likely to affect gender behavior and sexual orientation than gender identity $y^{20,21)}$.

The potential effects of prenatal and postnatal androgen exposure on gender identity outcome and "gender role change" have also been explored in other hormonal and nonhormonal $\operatorname{DSDs}^{22-26)}$. These studies indicate that gender identity is not solely dependent on prenatal and postnatal androgen exposure; however, the occurrence of gender identity change (in comparison to the "natal gender") at a rate significantly higher than would be expected in the general population supports some role of prenatal and possibly postnatal androgens in gender identity development. While the above-noted studies support some role of androgens in male gender identity, a case report in a 46, XY individual with Complete Androgen Insensitivity Syndrome challenges the concept that androgen receptor signaling is required for male gender identity development ${ }^{27}$. Potential limitations of all these survey/questionnaire-based studies to assess gender identity include a person's degree of self-awareness and one's willingness to disclose this information in the study context ${ }^{16)}$.

With respect to genetics and gender identity, heritability of transsexualism has been suggested primarily from studies describing concordance of transsexualism in monozygotic twin pairs ${ }^{28-311}$. A study supporting a role for genetic factors in gender identity outcome demonstrated a $39.1 \%$ concordance for GID (based on DSM-IV criteria) in 23 monozygotic female and male twin pairs, with no concordance in 21 same-sex dizygotic female and male twin pairs or in 7 opposite-sex twin pairs ${ }^{31}$. However, with respect to specific candidate genes, association studies of polymorphisms with transsexualism have been inconsistent and lacking strong statistical significance ${ }^{32-35)}$.

Regarding neuroanatomical considerations and gender identity, it has been known for some time that some brain 
structures are sexually dimorphic, while one component of the hypothalamus has been reported to be sexual orientationdimorphic $^{16)}$. Numerous studies in transgender adults, carried out prior to any cross-sex hormone treatment, have provided evidence indicating that some sexually dimorphic structures appear to align more closely with a person's gender identity than with their physical sex characteristics ${ }^{16)}$. A trend towards similar findings has also been reported in gray matter of gender dysphoric youth ${ }^{36)}$.

\section{Natural history of transgender youth}

The majority of gender dysphoric prepubertal youth will no longer meet the mental health criteria for gender dysphoria once puberty has begun, likely reflecting the heterogenous nature of this group ${ }^{2,7,8,37)}$. Investigators have attempted to identify factors that predict gender dysphoria "persisters" versus "desisters" ${ }^{138)}$. Persisters reported relatively greater degrees of gender dysphoria and were more likely to have experienced social transition (to their affirmed gender) during childhood ${ }^{38)}$. In addition, persisters believed they "were" the other sex, while desisters "wished they were" the other $\operatorname{sex}^{38)}$. The limitations in prediction of persistence, coupled with the observation that most gender dysphoric children will not become transgender adolescents or adults, have led some investigators and/or practitioners to promote efforts to encourage gender dysphoric children to accept their natal gender ${ }^{39)}$. In contrast, a model of care that "affirms" a child's gender expression is thought to have a more optimal mental health outcome $1,{ }^{40)}$. Long-term outcomes studies are needed to resolve these differences in approach to the care of gender dysphoric pre-pubertal youth.

\section{Multidisciplinary care of transgender youth}

Persistence of gender dysphoria following initiation of puberty implies a very high likelihood that such individuals will be transgender as adults; in fact, the emergence or worsening of gender dysphoria with onset of puberty is thought to have significant diagnostic value in the determination of being transgender ${ }^{41)}$. Given the complexity of these issues, it is essential that gender dysphoric youth undergo a thorough psychodiagnostic evaluation by a qualified mental health provider. An important role of the mental health/gender specialist is not only to determine the presence or absence of gender dysphoria, but to also evaluate for the presence of other mental health concerns. For example, while there is an increased association of gender dysphoria and autism spectrum disorder ${ }^{42,43)}$, the majority of gender dysphoric children and adolescents do not have an underlying severe psychiatric illness ${ }^{41)}$.

Based on pioneering work form the Netherlands, the Endocrine Society (ES) guidelines and WPATH SOC endorse the use of pubertal blockers using gonadotropin releasing hormone $(\mathrm{GnRH})$ agonists at Tanner stage II/III in individuals experiencing a significant increase in gender dysphoria with onset of puberty ${ }^{3,444-47)}$. This treatment is fully reversible and allows additional time for gender exploration without the pressure of ongoing pubertal development. The physical changes of puberty, once completed, cannot be reversed (by means other than surgical or, for voice, other than by voice training)-e.g., low voice, Adam's apple, and facial features in phenotypic males and breast development in phenotypic females. Preventing pubertal development that does not match a person's gender identity can theoretically lead to decreased distress, and can ultimately enable the individual to more easily "blend" in society as an adult. Subsequently, if the individual continues to identify as transgender, cross-sex hormones can be added while continuing GnRH agonist suppression of endogenous puberty, enabling the individual to experience only the physical changes of puberty that match the person's experienced/affirmed gender identity ${ }^{3,4)}$. While age-specific guidelines for subsequent interventions are not delineated in the WPATH SOC, the ES guidelines suggest that cross-sex hormones can be initiated at about the age of 16 years (the legal age for medical decision-making in some countries), while surgical procedures (with the exception of mastectomy) should be deferred until the individual is at least 18 years of age ${ }^{4)}$. Delaying cross-sex hormone treatment until age 16 could not only be detrimental to bone health, but, by keeping someone in a prepubertal state until this age, would isolate the individual further from age-matched peers, with potentially negative consequences for emotional well-being. Gender centers at our institution and elsewhere are therefore studying the impact of cross-sex hormone treatment initiation at 14 years of age (which approximates the upper end of the age range for normal pubertal onset in natal males and one year beyond the upper end of the age range in natal females). In this group, sex steroids are increased gradually over the course of $2-3$ years ${ }^{16}$.

There are currently only limited outcomes data to support the ES and WPATH recommendations for care of transgender youth. A report from the Netherlands assessed mental health outcomes in 55 transgender adolescents/young adults (22 MTF, 33 FTM) at 3-time points: Before the start of GnRH agonist treatment, at initiation of cross-sex hormones (average 16.7 years at start of treatment), and at least 1 year after "gender reassignment surgery" (average age 20.7 years) ${ }^{48)}$. Despite a decrease in depression and an improvement in general mental health functioning, gender dysphoria persisted during the period of pubertal suppression (as noted in earlier reports); however, following cross-sex hormone treatment and "gender reassignment surgery," gender dysphoria was resolved and psychological functioning steadily improved ${ }^{48)}$."Well-being" was found to be similar to or better than that in age-matched young adults from the general population, and none of the study participants regretted treatment ${ }^{48)}$. This study represents the first long-term follow-up of patients managed according to currently existing clinical practice guidelines for transgender youth, and underscores the benefit of the multidisciplinary approach to care.

The primary risks of pubertal suppression in gender dyspho- 
ric youth treated with GnRH agonists include adverse effects on bone mineralization, compromised fertility, and unknown effects on brain development.

A 6-year longitudinal, observational study assessed bone mineral density (BMD) in 34 transgender adolescents (15 MTF, 19 FTM) who had received GnRH agonist beginning at an average age of 14.9-15 years (individuals were mid-late pubertal at study onset by testicular volume or breast stage), had initiation of cross sex hormones at 16.4-16.6 years, followed by gonadectomy with discontinuation of GnRH agonist at a minimum age of 18 years ${ }^{49)}$. Over the 6-year observation period, areal BMD Z-scores decreased significantly in MTF individuals with a trend for a decrease in FTM individuals, suggesting either a delay in attainment of peak bone mass, or an attenuation of peak bone mass, itself $f^{49}$. As acknowledged by the authors, potential study limitations included a relatively small "n," the fact that individuals were already late pubertal at the time of GnRH agonist initiation, the possibility that relatively low doses of cross sex hormones were used during the initial period of that phase of treatment, and that information was not available regarding dietary calcium intake, Vitamin D levels, and weight bearing exercise, all of which can influence $\mathrm{BMD}^{49)}$. It would seem important, particularly during GnRH agonist treatment, to ensure adequate intake of calcium and Vitamin D, to encourage weight-bearing exercise, and to routinely monitor levels of 25 -
OH Vitamin $D^{16)}$.

Any use of pubertal blockers and cross-sex hormones in transgender youth should include an informed consent process and a discussion about implications for fertility. Transgender adolescents may wish to preserve fertility, which may be otherwise compromised if puberty is suppressed at an early stage and the patient completes phenotypic transition with the use of cross-sex hormones ${ }^{16)}$. While full in vitro maturation of germ cells has not yet been achieved in humans, promising studies have been carried out in mice $e^{50,51)}$.

Regarding potential adverse effects of GnRH agonists (and cross sex hormones) on brain, a recent study investigated whether GnRH agonist treatment of gender dysphoric adolescents would impair executive functioning (reasoning, problem solving, etc.), thought to reflect pre-frontal brain activation, and to have significant development during puberty ${ }^{52}$. No significant detrimental effects of GnRH agonist treatment on executive functioning were observed ${ }^{52)}$.

Some transgender adolescents first come to medical attention when they are late pubertal (Tanner stage IV/V). FTM individuals can be treated with testosterone alone while MTF individuals are optimally treated with estrogen with concurrent use of an agent that blocks testosterone secretion and/or action $^{3,4)}$. In this older cohort, cross sex hormone regimens may be increased to full replacement doses over a shorter interval

Table 1. Hormonal interventions for transgender adolescents (All currently off-label for gender nonconforming/transgender youth)

A. Inhibitors of gonadal sex steroid secretion or action

1. GnRH analogues: Inhibition of the hypothalamic-pituitary-gonadal (HPG) axis (FTM and MTF)

a. Leuprolide acetate IM (1- or 3-month preparations) or SC (1-, 3-, 4-, or 6-month preparations) at dose sufficient to suppress pituitary gonadotropins and gonadal sex steroids

b. Histrelin acetate SC implant (once-yearly dosing, though may have longer effectiveness)

c. Other options: goserelin acetate SC implant (4- or 12-week preparations); nafarelin acetate Intranasal (multiple daily doses) also available, but no reported use in this population

2. Alternative approaches

a. Medroxyprogesterone acetate orally (up to $40 \mathrm{mg} /$ day) or IM (150 mg q 3 months): Inhibition of HPG axis and direct inhibition of gonadal steroidogenesis (FTM an MTF)

b. Spironolactone (25 to $50 \mathrm{mg} /$ day with gradual increase to $100-300 \mathrm{mg} /$ day orally, divided into BID dosing): Inhibition of testosterone synthesis and action (MTF)

c. Cyproterone acetate (gradual increase up to $100 \mathrm{mg} /$ day orally; not available in U.S.):

Inhibition of testosterone synthesis and action (MTF)

d. Finasteride (2.5-5 mg/day orally)

Inhibition of type II 5 a-reductase, blocking conversion of testosterone to 5 a-dihydrotestosterone (MTF)

B. Cross-sex hormones

1. MTF: Estrogen: $17 \beta$-estradiol

a. Transdermal: twice weekly patches ( $6.25 \mathrm{mcg}$ [achieved by cutting a $25 \mathrm{mcg}$ patch] with gradual increase to full adult dose)

b. Oral/sublingual: daily $(0.25 \mathrm{mg}$ with gradual increase to full adult dose of $2-6 \mathrm{mg} /$ day)

c. Parenteral IM (synthetic esters of 17 -estradiol): estradiol valerate (5-20 mg up to 30-40 mg q 2 weeks) or estradiol cypionate (2-10 mg q 1 week)

2. FTM: Testosterone

a. Parenteral IM or SC (synthetic esters of testosterone): testosterone cypionate or enanthate (12.5 mg q week or 25 mg q 2 weeks, with gradual increase to 50-100 mg q week or 100-200 mg q 2 weeks)

b. Transdermal (consider once full adult testosterone dose has been achieved parenterally): patch (2.5-7.5 mg/day) or $1 \%$ gel (2.5-10 grams/day of gel $=25-100 \mathrm{mg} /$ day of testosterone)

GnRH, gonadotropin releasing hormone; IM, intramuscular; SC, subcutaneous; FTM: female-to-male; MTF, male-to-female; q, every.

Adapted from Rosenthal SM. J Clin Endocrinol Metab 2014;99:4379-89, with permission of .the Endocrine Society ${ }^{16}$. 
than used for the younger cohort that had been initially treated with pubertal blockers at Tanner II/III ${ }^{16)}$. While GnRH agonists are the preferred option for pubertal suppression in both the early and late pubertal individuals, this treatment is costly and often inaccessible ${ }^{16)}$. Options for pubertal suppression and cross sex hormone treatment are listed in Table $1^{16)}$. With respect to estrogen treatment, $17 \beta$-estradiol (transdermal, oral, or parenteral) is preferred to conjugated (e.g., premarin) or synthetic estrogens (e.g., ethinyl estradiol) given that conjugated and synthetic estrogen levels cannot be monitored in the serum and that ethinyl estradiol (in comparison to $17 \beta$-estradiol) is associated with an increased risk for venous thromboembolic disease and death from cardiovascular causes ${ }^{53,54)}$. Table $2^{16)}$ summarizes surveillance recommendations for desired as well as adverse effects during treatment with pubertal blockers alone and in combination with cross-sex hormones, adapted from the current ES guidelines ${ }^{4)}$.

Some Tanner stage IV/V-transgender adolescents present for medical services before 16 years of age. As with the group treated with GnRH agonists at early puberty, we and others are studying the consequences of cross-sex hormone treatment at 14 years of age ${ }^{16)}$. Some gender dysphoric youth first come to medical attention when they are Tanner stage IV/V, but $<14$ years of age. These individuals would be candidates for pubertal blockers (e.g., to stop menses in an FTM adolescent), but without supportive outcomes data, not currently candidates for cross-sex hormone use under most circumstances ${ }^{16)}$.

\section{Challenges to optimal care and priorities for research}

Areas of uncertainty and barriers to state-of-the art practice limit the ability to provide optimal health care to gender dysphoric/transgender youth. Currently, there are only limited safety and efficacy studies, with virtually no published data on the use of pubertal blockers in gender dysphoric individuals $<12$ years of age or cross-sex hormones in transgender youth $<16$ years of age. In addition, randomized controlled trials for hormonal interventions in gender dysphoric youth have not been considered feasible or ethical ${ }^{55}$. Current clinical practice guidelines are based on best available evidence, with significant reliance on expert opinion. While an increasing number of clinical programs have emerged around the world in recent years, there are many geographic regions in which such services do not exist, limiting access to care and often requiring patients and families to travel long distances. Furthermore, lack of training of providers and prejudice and misunderstanding on the part of family, community, and medical and mental health professionals may limit access to optimal care ${ }^{56)}$.

\section{Conclusions}

While significant advances in our understanding of gender nonconforming/transgender youth have been achieved, many gaps in knowledge remain. Compelling studies have demonstrated that gender identity is not simply a psychosocial construct, but likely reflects a complex interplay of biologic, environmental, and cultural factors ${ }^{16)}$. The replacement of "disorder" with "dysphoria" in DSM-5 removes the connotation that a transgender identity, itself, is pathologic, and instead, focuses on dysphoria as the clinical concern ${ }^{2}$. A landmark study from the Netherlands indicates that mental health comorbidities in gender-dysphoric youth significantly diminish or resolve when such individuals are subject to a gender-affirming

Table 2. Monitoring during pubertal suppression and during cross-sex hormone treatment*

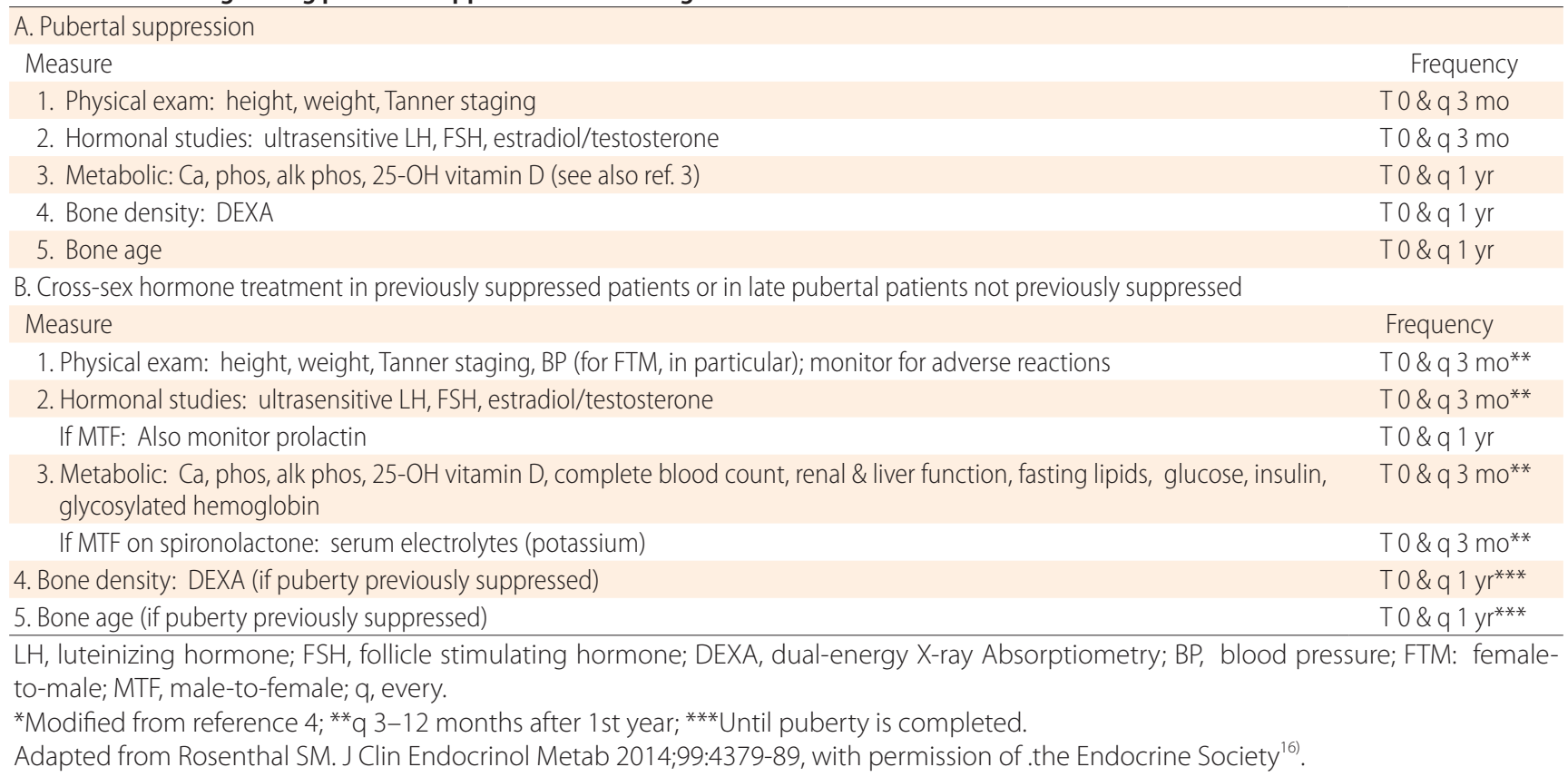


model of care, optimally delivered in a multi-disciplinary clinical setting ${ }^{48}$. Further prospective studies focused on longterm safety and efficacy are necessary to optimize medical and mental health care for transgender youth.

\section{Conflict of interest}

The author has served as a consultant to AbbVie. Inc. Except for that, no potential conflict of interest relevant to this article was reported.

\section{Acknowledgments}

This work is supported, in part, by a grant for the National Institutes of Health: "The Impact of Early Medical Treatment in Transgender Youth" (1R01HD082554-01A1). I gratefully acknowledge the generous invitation of the Korean Society of Pediatric Endocrinology (KSPE) to have participated in the 20th Anniversary meeting of KSPE.

\section{References}

1. Vance SR Jr, Ehrensaft D, Rosenthal SM. Psychological and medical care of gender nonconforming youth. Pediatrics 2014;134:1184-92.

2. American Psychiatric Association. Diagnostic and statistical manual of mental disorders. 5th ed. Arlington (VA); American Psychiatric Association Publishing; 2013.

3. Coleman E, Bockting W, Botzer M, Cohen-Kettenis P, DeCuypere G, Feldman J, et al. Standards of care for the health of transsexual, transgender, and gendernonconforming people, version 7. Int J Transgenderism 2012;13:165-232.

4. Hembree WC, Cohen-Kettenis P, Delemarre-van de Waal HA, Gooren LJ, Meyer WJ 3rd, Spack NP, et al. Endocrine treatment of transsexual persons: an Endocrine Society clinical practice guideline. J Clin Endocrinol Metab 2009;94:3132-54.

5. Meyer-Bahlburg HF. Sex steroids and variants of gender identity. Endocrinol Metab Clin North Am 2013;42:435-52.

6. Sherer I, Baum J, Ehrensaft D, Rosenthal SM. Affirming gender: caring for gender atypical children and adolescents. Contemp Pediatr 2015;32:16-9.

7. Drummond KD, Bradley SJ, Peterson-Badali M, Zucker KJ. A follow-up study of girls with gender identity disorder. Dev Psychol 2008;44:34-45.

8. Wallien MS, Cohen-Kettenis PT. Psychosexual outcome of gender-dysphoric children. J Am Acad Child Adolesc Psychiatry 2008;47:1413-23.

9. Zucker KJ, Bradley SJ, Owen-Anderson A, Kibblewhite SJ, Cantor JM. Is gender identity disorder in adolescents coming out of the closet? J Sex Marital Ther 2008;34:28790.

10. de Vries AL, Cohen-Kettenis PT. Clinical management of gender dysphoria in children and adolescents: the Dutch approach. J Homosex 2012;59:301-20.

11. Spack NP, Edwards-Leeper L, Feldman HA, Leibowitz S, Mandel F, Diamond DA, et al. Children and adolescents with gender identity disorder referred to a pediatric medical center. Pediatrics 2012;129:418-25.

12. Sherer I, Rosenthal SM, Ehrensaft D, Baum J. Child and adolescent gender center: a multidisciplinary collaboration to improve the lives of gender nonconforming children and teens. Pediatr Rev 2012;33:273-5.

13. Conron KJ, Scott G, Stowell GS, Landers SJ. Transgender health in Massachusetts: results from a household probability sample of adults. Am J Public Health 2012;102:118-22.

14. Reisner SL, Vetters R, Leclerc M, Zaslow S, Wolfrum S, Shumer D, et al. Mental health of transgender youth in care at an adolescent urban community health center: a matched retrospective cohort study. J Adolesc Health 2015;56:274-9.

15. Travers R, Bauer G, Pyne J, Bradley K, for the Trans PULSE Project; Gale L, et al: Impacts of strong parental support for trans youth: a report prepared for Children'S Aid Society of Toronto and Delisle Youth Services. Trans Pulse 2012 Oct 2.

16. Rosenthal SM. Approach to the patient: transgender youth: endocrine considerations. J Clin Endocrinol Metab 2014;99:4379-89.

17. Berenbaum SA, Bailey JM. Effects on gender identity of prenatal androgens and genital appearance: evidence from girls with congenital adrenal hyperplasia. J Clin Endocrinol Metab 2003;88:1102-6.

18. Dessens AB, Slijper FM, Drop SL. Gender dysphoria and gender change in chromosomal females with congenital adrenal hyperplasia. Arch Sex Behav 2005;34:389-97.

19. Meyer-Bahlburg HF, Dolezal C, Baker SW, Ehrhardt AA, New MI. Gender development in women with congenital adrenal hyperplasia as a function of disorder severity. Arch Sex Behav 2006;35:667-84.

20. Frisén L, Nordenström A, Falhammar H, Filipsson H, Holmdahl G, Janson PO, et al. Gender role behavior, sexuality, and psychosocial adaptation in women with congenital adrenal hyperplasia due to CYP21A2 deficiency. J Clin Endocrinol Metab 2009;94:3432-9.

21. Meyer-Bahlburg HF, Dolezal C, Baker SW, New MI. Sexual orientation in women with classical or non-classical congenital adrenal hyperplasia as a function of degree of prenatal androgen excess. Arch Sex Behav 2008;37:85-99.

22. Cohen-Kettenis PT. Gender change in $46, \mathrm{XY}$ persons with 5alpha-reductase-2 deficiency and 17beta-hydroxysteroid dehydrogenase-3 deficiency. Arch Sex Behav 2005;34:399410 .

23. Imperato-McGinley J, Peterson RE, Gautier T, Sturla E. Androgens and the evolution of male-gender identity among male pseudohermaphrodites with 5alpha-reductase deficiency. N Engl J Med 1979;300:1233-7.

24. Rösler A, Silverstein S, Abeliovich D. A (R80Q) mutation in 17 beta-hydroxysteroid dehydrogenase type 3 gene among Arabs of Israel is associated with pseudohermaphroditism 
in males and normal asymptomatic females. J Clin Endocrinol Metab 1996;81:1827-31.

25. Reiner WG, Gearhart JP. Discordant sexual identity in some genetic males with cloacal exstrophy assigned to female sex at birth. N Engl J Med 2004;350:333-41.

26. Meyer-Bahlburg HF. Gender identity outcome in femaleraised 46, XY persons with penile agenesis, cloacal exstrophy of the bladder, or penile ablation. Arch Sex Behav 2005;34:423-38.

27. T'Sjoen G, De Cuypere G, Monstrey S, Hoebeke P, Freedman FK, Appari M, et al. Male gender identity in complete androgen insensitivity syndrome. Arch Sex Behav 2011;40:635-8.

28. Hyde C, Kenna JC. A male MZ twin pair, concordant for transsexualism, discordant for schizophrenia. Acta Psychiatr Scand 1977;56:265-75.

29. Green R. Family cooccurrence of "gender dysphoria": ten sibling or parent-child pairs. Arch Sex Behav 2000;29:499507.

30. Coolidge FL, Thede LL, Young SE. The heritability of gender identity disorder in a child and adolescent twin sample. Behav Genet 2002;32:251-7.

31. Heylens G, De Cuypere G, Zucker KJ, Schelfaut C, Elaut E, Vanden Bossche H, et al. Gender identity disorder in twins: a review of the case report literature. J Sex Med 2012;9:7517.

32. Henningsson S, Westberg L, Nilsson S, Lundström B, Ekselius L, Bodlund O, et al. Sex steroid-related genes and male-to-female transsexualism. Psychoneuroendocrinology 2005;30:657-64.

33. Hare L, Bernard P, Sánchez FJ, Baird PN, Vilain E, Kennedy $\mathrm{T}$, et al. Androgen receptor repeat length polymorphism associated with male-to-female transsexualism. Biol Psychiatry 2009;65:93-6.

34. Ujike H, Otani K, Nakatsuka M, Ishii K, Sasaki A, Oishi T, et al. Association study of gender identity disorder and sex hormone-related genes. Prog Neuropsychopharmacol Biol Psychiatry 2009;33:1241-4.

35. Bentz EK, Hefler LA, Kaufmann U, Huber JC, Kolbus A, Tempfer CB. A polymorphism of the CYP17 gene related to sex steroid metabolism is associated with female-tomale but not male-to-female transsexualism. Fertil Steril 2008;90:56-9.

36. Hoekzema E, Schagen SE, Kreukels BP, Veltman DJ, CohenKettenis PT, Delemarre-van de Waal H, et al. Regional volumes and spatial volumetric distribution of gray matter in the gender dysphoric brain. Psychoneuroendocrinology 2015;55:59-71.

37. Zucker KJ. On the "natural history" of gender identity disorder in children. J Am Acad Child Adolesc Psychiatry 2008;47:1361-3.

38. Steensma TD, McGuire JK, Kreukels BP, Beekman AJ, Cohen-Kettenis PT. Factors associated with desistence and persistence of childhood gender dysphoria: a quantitative follow-up study. J Am Acad Child Adolesc Psychiatry 2013;52:582-90.
39. Zucker KJ, Wood H, Singh D, Bradley SJ. A developmental, biopsychosocial model for the treatment of children with gender identity disorder. J Homosex 2012;59:369-97.

40. Hidalgo MA, Ehrensaft D, Tishelman AC, Clark LF, Garofalo R, Rosenthal SM, et al. The gender affirmative model: what we know and what we aim to learn. Hum Dev 2013;56:285-90.

41. Cohen-Kettenis PT, Delemarre-van de Waal HA, Gooren LJ. The treatment of adolescent transsexuals: changing insights. J Sex Med 2008;5:1892-7.

42. de Vries AL, Noens IL, Cohen-Kettenis PT, van BerckelaerOnnes IA, Doreleijers TA. Autism spectrum disorders in gender dysphoric children and adolescents. J Autism Dev Disord 2010;40:930-6.

43. Strang JF, Kenworthy L, Dominska A, Sokoloff J, Kenealy $\mathrm{LE}$, Berl M, et al. Increased gender variance in autism spectrum disorders and attention deficit hyperactivity disorder. Arch Sex Behav 2014;43:1525-33.

44. Cohen-Kettenis PT, van Goozen SH. Pubertal delay as an aid in diagnosis and treatment of a transsexual adolescent. Eur Child Adolesc Psychiatry 1998;7:246-8.

45. Delemarre-van de Waal HA, Cohen-Kettenis PT. Clinical management of gender identity disorder in adolescents: a protocol on psychological and paediatric endocrinology aspects. Eur J Endocrinol 2006;155:S131-7.

46. de Vries AL, Steensma TD, Doreleijers TA, Cohen-Kettenis PT. Puberty suppression in adolescents with gender identity disorder: a prospective follow-up study. J Sex Med 2011;8:2276-83.

47. Cohen-Kettenis PT, Schagen SE, Steensma TD, de Vries AL, Delemarre-van de Waal HA. Puberty suppression in a gender-dysphoric adolescent: a 22-year follow-up. Arch Sex Behav 2011;40:843-7.

48. de Vries AL, McGuire JK, Steensma TD, Wagenaar EC, Doreleijers TA, Cohen-Kettenis PT. Young adult psychological outcome after puberty suppression and gender reassignment. Pediatrics 2014;134:696-704.

49. Klink D, Caris M, Heijboer A, van Trotsenburg M, Rotteveel J. Bone mass in young adulthood following gonadotropinreleasing hormone analog treatment and cross-sex hormone treatment in adolescents with gender dysphoria. J Clin Endocrinol Metab 2015;100:E270-5.

50. Sato T, Katagiri K, Gohbara A, Inoue K, Ogonuki N, Ogura $\mathrm{A}$, et al. In vitro production of functional sperm in cultured neonatal mouse testes. Nature 2011;471:504-7.

51. Yokonishi T, Sato T, Komeya M, Katagiri K, Kubota Y, Nakabayashi K, et al. Offspring production with sperm grown in vitro from cryopreserved testis tissues. Nat Commun 2014;5:4320.

52. Staphorsius AS, Kreukels BP, Cohen-Kettenis PT, Veltman DJ, Burke SM, Schagen SE, et al. Puberty suppression and executive functioning: an fMRI-study in adolescents with gender dysphoria. Psychoneuroendocrinology 2015;56:190-9.

53. Toorians AW, Thomassen MC, Zweegman S, Magdeleyns EJ, Tans G, Gooren LJ, et al. Venous thrombosis and 
changes of hemostatic variables during cross-sex hormone treatment in transsexual people. J Clin Endocrinol Metab 2003;88:5723-9.

54. Asscheman H, Giltay EJ, Megens JA, de Ronde WP, van Trotsenburg MA, Gooren LJ. A long-term follow-up study of mortality in transsexuals receiving treatment with crosssex hormones. Eur J Endocrinol 2011;164:635-42.
55. Drescher J, Byne W. Gender dysphoric/gender variant (GD/ GV) children and adolescents: summarizing what we know and what we have yet to learn. J Homosex 2012;59:501-10.

56. Vance SR Jr, Halpern-Felsher BL, Rosenthal SM. Health care providers' comfort with and barriers to care of transgender youth. J Adolesc Health 2015;56:251-3. 